\title{
Some New Results on Equal Sums of Like Powers
}

\author{
By Simcha Brudno
}

Abstract. The Diophantine equation $\sum_{i=1}^{M} x_{i}{ }^{n}=\sum_{i=1}^{M} y_{i}{ }^{n}$ is examined for $n=3,4$ and 6 and $M=[(n+1) / 2]$. A method for generating pe rametric solutions for $n=4$ is derived and several new numerical examples for $n=4,6$ are given. The method also applies for all other values of $M$ and possibly for values of $n$ greater than 6 , too.

1. In this article we describe a method to get many integral solutions of the type

$$
\sum_{i=1}^{M} x_{i}^{n}=\sum_{i=1}^{M} y_{i}^{n}
$$

from one known solution. While this method is general for any $M$, this article will be limited to cases where $M=[(n+1) / 2]$.

For the case $n=3$, that is, $M=2$, the equation becomes:

$$
x_{1}{ }^{3}+x_{2}{ }^{3}=y_{1}{ }^{3}+y_{2}{ }^{3} \text {. }
$$

We solve the system of linear equations:

$$
\begin{aligned}
& p_{1}+q_{1}=x_{1}, \quad p_{2}+q_{1}=y_{1}, \\
& p_{2}+q_{2}=x_{2}, \quad p_{1}-q_{2}=y_{2}
\end{aligned}
$$

for $p_{i}$ and $q_{i}$. In general the system is characterized by the equations

$$
\begin{aligned}
p_{i}+q_{i}=x_{i}, & i=1 \cdots M, \\
p_{i+1}+q_{i}=y_{i}, & i=1 \cdots M-1
\end{aligned}
$$

and

$$
p_{1}-q_{M}=y_{M} .
$$

This last equation is included to make the determinant nonzero and thereby guarantee unique rational $p_{i}{ }^{\prime}$ s and $q_{i}{ }^{\prime}$ s from each numerical set of $x_{i}{ }^{\prime}$ s and $y_{i}{ }^{\prime}$ 's.

Next we develop the equations:

$$
\sum_{i=1}^{M}\left(p_{i}+\lambda q_{i}\right)^{n}-\sum_{i=1}^{M-1}\left(p_{i+1} \lambda+q_{i}\right)^{n}-\left(p_{1}-\lambda q_{M}\right)^{n}=0 .
$$

We arrive at polynomials of the $n$th degree in $\lambda$. Because the $p_{i}{ }^{n}$ 's always cancel and the $q_{i}{ }^{n}$ cancel whenever $n$ is even, we are left with a polynomial of one degree lower for odd $n$ and two degrees lower for even $n$ in $\lambda$. We also know that the same polynomial has a solution $\lambda=1$ which, when substituted gives us our initial numerical example:

$$
\sum_{1}^{M} x_{i}{ }^{n}=\sum_{1}^{M} y_{i}{ }^{n}
$$

Received July 23, 1968, revised January 15, 1969. 
Therefore we factor out $(\lambda-1)$ and are left with a polynomial in $\lambda$ which is two degrees lower than the original equation in the case of $n$ odd and three degrees lower in the case of $n$ even. If one of the roots of the remaining polynomial in $\lambda$ is rational, it can then be used in Eq. (10) to generate a new numerical example.

For example, for the cases $n=3$ and $n=4$, this method is sufficient to give us another numerical example from any initial case because we are left with a linear equation in $\lambda$. Since we can interchange the $x_{i}$ in an even polynomial with $-x_{i}$, and in an odd polynomial with $-y_{i}$, we obtain many more numerical examples from a given one, which might or might not coincide.

From the equation of the third order: $3^{3}+4^{3}=-5^{3}+6^{3}$ we obtain twelve numerical examples:

$$
\begin{aligned}
& (-38,87,79,48), \\
& (18,19,28,-21), \\
& (-177,406,343,276), \\
& (-162,229,157,192), \\
& (-65,156,142,87), \\
& (15,-2,16,-9)
\end{aligned}
$$

and the other six degenerate to the initial case.

In the case of $n=4$, we take as our initial example

$$
133^{4}+134^{4}=59^{4}+158^{4}
$$

and obtain the following eight other numerical examples:

$$
\begin{array}{rlrl}
12505169907^{4}+783453422^{4} & =7038985479^{4}+12178821457^{4} \\
1^{4}+\quad 2^{4} & = & 2^{4}+ & 1^{4}
\end{array}
$$

$\begin{array}{rrrr}1^{4}+ & 2^{4}= & 2^{4}+ & 1^{4} \\ 111637^{4}+ & 114613^{4}= & 34813^{4}+ & 134413^{4} \\ 3687711^{4}+ & 6565526^{4}= & 1967986^{4}+ & 6710751^{4} \\ 1137493^{4}+ & 654854^{4}= & 1167518^{4}+ & 60779^{4} \\ 10381^{4}+ & 10203^{4}= & 2903^{4}+ & 12231^{4} \\ 1453319^{4}+ & 829418^{4}= & 461882^{4}+ & 1486969^{4} \\ 1054067^{4}+ & 545991^{4}= & 1057167^{4}+ & 522059^{4} .\end{array}$

2. When $x_{i}$ and $y_{i}$ are functions of a parameter, i.e., in the case where we start with a general two parametric formula for the solution of Eq. (1) the method described in Section 1 can also be used to obtain additional general formulas for the solutions. For example [4]:

$$
\begin{aligned}
& x_{1}=a^{7}+a^{5} b^{2}-2 a^{3} b^{4}+3 a^{2} b^{5}+a b^{6} \\
& x_{2}=a^{6} b-3 a^{5} b^{2}-2 a^{4} b^{3}+a^{2} b^{5}+b^{7} \\
& y_{1}=a^{7}+a^{5} b^{2}-2 a^{3} b^{4}-3 a^{2} b^{5}+a b^{6} \\
& y_{2}=a^{6} b+3 a^{5} b^{2}-2 a^{4} b^{3}+a^{2} b^{5}+b^{7} .
\end{aligned}
$$

From this, if we now define $p$ 's and $q$ 's as in Section 1 and solve for the $\lambda$ 's in terms 
of $a$ 's and $b$ 's, we obtain the following four formulas:

$$
\begin{aligned}
f(a, b)_{1}= & a \\
f(a, b)_{2}= & -a^{13}+a^{12} b+a^{11} b^{2}+5 a^{10} b^{3}+6 a^{9} b^{4}-12 a^{8} b^{5}-4 a^{7} b^{6} \\
& +7 a^{6} b^{7}-3 a^{5} b^{8}-3 a^{4} b^{9}+4 a^{3} b^{10}+2 a^{2} b^{11}-a b^{12}+b^{13} \\
f(a, b)_{3}= & a^{19}+6 a^{17} b^{2}-18 a^{15} b^{4}+6 a^{14} b^{5}-5 a^{13} b^{6}+12 a^{12} b^{7} \\
& -12 a^{11} b^{8}+36 a^{10} b^{9}-24 a^{9} b^{10}-12 a^{8} b^{11}+19 a^{7} b^{12} \\
& +36 a^{6} b^{13}+6 a^{5} b^{14}+12 a^{4} b^{15}-6 a^{3} b^{16}+6 a^{2} b^{17}+a b^{18} \\
f(a, b)_{4}= & a^{31}-a^{30} b+11 a^{29} b^{2}+a^{28} b^{3}+42 a^{27} b^{4}+24 a^{26} b^{5} \\
& -19 a^{25} b^{6}-32 a^{24} b^{7}-154 a^{23} b^{8}-254 a^{22} b^{9}+266 a^{21} b^{10} \\
& +718 a^{20} b^{11}+126 a^{19} b^{12}-303 a^{18} b^{13}-478 a^{17} b^{14} \\
& -830 a^{16} b^{15}+770 a^{15} b^{16}+916 a^{14} b^{17}-738 a^{13} b^{18} \\
& +21 a^{12} b^{19}+350 a^{11} b^{20}-434 a^{10} b^{21}+50 a^{9} b^{22}+142 a^{8} b^{23} \\
& -91 a^{7} b^{24}+76 a^{6} b^{25}+15 a^{5} b^{26}-3 a^{4} b^{27}+8 a^{3} b^{28} \\
& -8 a^{2} b^{29}+a b^{30}-b^{31}
\end{aligned}
$$

where $x_{1}=f(a, b)_{n}, x_{2}=f(b,-a)_{n}, y_{1}=f(a,-b)_{n}$ and $y_{2}=f(b, a)_{n}$. For the numerical values $a=2, b=1$, Eqs. (21), (22), (23), and (24), give the numerical examples (12), (16), (17), and (11) respectively.

It is interesting to note that all these four formulas are of the power $6 n+1$.

The other four numerical examples are given by the following formula:

$$
\begin{aligned}
x_{1}= & a^{18} b+3 a^{17} b^{2}-15 a^{16} b^{3}+15 a^{15} b^{4}+6 a^{14} b^{5}-45 a^{13} b^{6}+82 a^{12} b^{7} \\
& -15 a^{11} b^{8}-123 a^{10} b^{9}+171 a^{9} b^{10}-159 a^{8} b^{11}+159 a^{7} b^{12}-98 a^{6} b^{13} \\
& +30 a^{5} b^{14}-12 a^{4} b^{15}+3 a^{2} b^{17}+b^{19} \\
x_{2}= & a^{19}-a^{18} b-3 a^{17} b^{2}-3 a^{16} b^{3}+21 a^{15} b^{4}-12 a^{14} b^{5}-44 a^{13} b^{6} \\
& +86 a^{12} b^{7}-93 a^{11} b^{8}+87 a^{10} b^{9}+3 a^{9} b^{10}-135 a^{8} b^{11}+142 a^{7} b^{12} \\
& -100 a^{6} b^{13}+72 a^{5} b^{12}-36 a^{4} b^{15}+12 a^{3} b^{16}-9 a^{2} b^{17}+a b^{18}-b^{19} \\
y_{1}= & a^{19}-a^{18} b-3 a^{17} b^{2}-3 a^{16} b^{3}+21 a^{15} b^{4}-6 a^{14} b^{5}-44 a^{13} b^{6} \\
& +62 a^{12} b^{7}+15 a^{11} b^{8}-129 a^{10} b^{9}+165 a^{9} b^{10}-129 a^{8} b^{11}+88 a^{7} b^{12} \\
& -46 a^{6} b^{13}+18 a^{5} b^{14}-6 a^{4} b^{15}+12 a^{3} b^{16}-3 a^{2} b^{17}+a b^{18}-b^{19} \\
y_{2}= & a^{18} b-3 a^{17} b^{2}+3 a^{16} b^{3}+21 a^{15} b^{4}-60 a^{14} b^{5}+27 a^{13} b^{6}+58 a^{12} b^{7} \\
& -75 a^{11} b^{8}+57 a^{10} b^{9}-63 a^{9} b^{10}+63 a^{8} b^{11}-87 a^{7} b^{12}+100 a^{6} b^{13} \\
& -66 a^{5} b^{14}+36 a^{4} b^{15}-18 a^{3} b^{16}+9 a^{2} b^{17}+b^{19} .
\end{aligned}
$$

For the values $a=2, b=1 ; a=-2, b=1 ; a=1, b=2 ; a=1, b=-2$, Eq. (25) gives the numerical examples (13), (14), (15) and (18). The formulas (22) and (25) have been given by Lander [3] previously.

3. For the case $n=6, m=3$, the equation is of the third order and therefore 
has at least one real solution. This real solution need not be rational. Rational solutions to the $\lambda$ equation are found by a trial factoring method.

By factoring the $\lambda$ polynomial and taking the first known example:

$$
23^{6}+( \pm 10)^{6}+( \pm 15)^{6}=( \pm 3)^{6}+( \pm 19)^{6}+( \pm 22)^{6}
$$

we obtain eighteen new solutions, sixteen of which are trivial solutions of the form $a^{6}+b^{6}+c^{6}=( \pm a)^{6}+( \pm b)^{6}+( \pm c)^{6}$ and their permutations.

The remaining two are:

$$
81^{6}+50^{6}+37^{6}=65^{6}+78^{6}+11^{6}
$$

and

$$
32^{6}+43^{6}+81^{6}=3^{6}+55^{6}+80^{6} \text {. }
$$

Other solutions, which do not seem to have been previously recorded, obtained by the same method, are:

$$
\begin{aligned}
275^{6}+36^{6}+179^{6} & =65^{6}+276^{6}+169^{6} \\
211^{6}+125^{6}+300^{6} & =68^{6}+289^{6}+249^{6} \\
1^{6}+515^{6}+500^{6} & =556^{6}+197^{6}+409^{6} \\
148^{6}+249^{6}+103^{6} & =188^{6}+243^{6}+11^{6} \\
539^{6}+412^{6}+643^{6} & =497^{6}+652^{6}+449^{6} .
\end{aligned}
$$

Attempts to find a parametric expression for $n>6$ have thus far been fruitless.

Massachusetts Institute of Technology

Cambridge, Massachusetts 02139

1. E. Fauquembergue, L'Intermédiare des Mathématiciens, v. 21, 1914, p. 17.

2. L. Euler, Novi Commentarii Acad. Petropol., v. 17, 1772, p. 64.

3. L. J. LANDER, "Geometric aspects of Diophantine equations involving equal sums of like powers," Amer. Math. Monthly, v. 75, 1968, p. 1061.

4. A. Gerardin, L'Intermédiaire des Mathématiciens, v. 24, 1917, p. 51.

5. K. SubBa RAO, "On sums of sixth powers," J. London Math. Soc., v. 9, 1934, p. 173.

6. L. J. Lander, T. R. Parkin \& J. L. Selfridge, "A survey of equal sums of like powers," Math. Comp., v. 21, 1967, pp. 446-459. MR $36 \# 5060$. 